\title{
El Mini Mental State Examination no es la única prueba cognitiva útil para la detección de demencia
}

\author{
Mini Mental State Examination is not the only cognitive test useful for detection of dementia
}

\section{Objetivos}

Evaluar la exactitud diagnóstica de diferentes pruebas cognitivas para la detección de la demencia mediante una revisión sistemática de la literatura.

\section{Fuentes de datos y selección de estudios}

MEDLINE, EMBASE, PsychoINFO, Google Scholar (hasta septiembre 2014) y listado de referencias de los artículos incluidos. Se incluyeron estudios enfocados en la identificación de pacientes con demencia asociada a la enfermedad de Alzheimer, demencia vascular o enfermedad de Parkinson en cualquier contexto (clínico o comunitario), que reportaran la sensibilidad y especificidad de las pruebas de tamizaje evaluadas (aquellas con tiempo de administración igual o inferior a 20 minutos) y que usaran criterios estándar para el diagnóstico de demencia. Se excluyeron artículos en idiomas diferentes al
Tsoi K, y cols. JAMA Internal Medicine 2015;175(9):1450-8. inglés, estudios referentes a pruebas que no sumaran más de cuatro referencias o estudios en pacientes con discapacidad visual.

\section{Extracción de datos}

Dos autores evaluaron de manera independiente las referencias, seleccionando los estudios elegibles y haciendo la posterior extracción de datos por medio de un formato preestablecido. Se evaluó el riesgo de sesgo de cada estudio por medio del instrumento QUADAS-2, presentando como resumen el porcentaje de alto riesgo de sesgo. Asimismo, se diseñó una escala de ocho puntos para expresar cuantitativamente la calidad general de los estudios para cada prueba evaluada. Para el análisis de datos se usó el procedimiento METANDI y MIDAS en STATA 11, ajustando un modelo bivariado de efectos aleatorios. La heterogeneidad fue evaluada por medio del estadístico I2.

Resultados principales. Tabla 1. Meta-análisis de la exactitud diagnóstica de las cinco principales pruebas de tamizaje de demencia.

\begin{tabular}{l|c|c|c|c} 
Prueba de tamizaje & $\begin{array}{c}\text { Número de estudios } \\
\text { incluidos }\end{array}$ & $\begin{array}{c}\text { Sensibilidad agrupada } \\
\text { (IC 95\%) }\end{array}$ & $\begin{array}{c}\text { Especificidad agrupada } \\
\text { (IC 95\%) }\end{array}$ & $\begin{array}{c}\text { Mediana de la calidad de los } \\
\text { estudios (Rango) }\end{array}$ \\
\hline ACE-R & 13 & $0,92(0,90 \mathrm{a} 0,94)$ & $0,89(0,84 \mathrm{a} \mathrm{0,93)}$ & $7(6 \mathrm{a} 8)$ \\
\hline Mini-Cog & 9 & $0,91(0,80 \mathrm{a} 0,96)$ & $0,86(0,74 \mathrm{a} \mathrm{0,93)}$ & $7(5 \mathrm{a} 8)$ \\
\hline MoCA & 20 & $0,91(0,84 \mathrm{a} 0,95)$ & $0,81(0,71 \mathrm{a} 0,88)$ & $8(4 \mathrm{a} 8)$ \\
\hline GPCOG & 5 & $0,92(0,81 \mathrm{a} 0,97)$ & $0,87(0,83 \mathrm{a} 0,90)$ & $7,5(4 \mathrm{a} 8)$ \\
\hline MMSE & 108 & $0,81(0,78 \mathrm{a} 0,84)$ & $0,89(0,87 \mathrm{a} 0.91)$ & $7(3 \mathrm{a} 8)$ \\
\hline
\end{tabular}

ACE-R: Addenbrooke's Cognitive Examination Revised. MoCA: Montreal Cognitive Assessment. GPCOG: General Practitioner Assessment of Cognition. MMSE: Mini-Mental State Examination.

\section{Conclusiones}

Además del MMSE, existen otras pruebas con similar capacidad diagnóstica para la detección de demencia. El Mini-Cog y el ACE-R son las mejores alternativas para el tamizaje de de- mencia, y el MOCA es la mejor alternativa para la detección del deterioro cognitivo leve (DCL).

Fuente de financiamiento: no referida.

\section{Comentario}

La revisión de Tsoi y colaboradores resume la evidencia de un controvertido tópico como es el tamizaje de demencia en población adulta mayor. Las cifras de demencia han aumentado considerablemente con la esperanza de vida, y si bien no se cuenta en la actualidad con tratamientos que "curen" la enfermedad, un diagnóstico oportuno puede traer beneficios personales y sociales de importancia para los adultos con esta condición. La revisión de Tsoi se ha desarrollado bajo las metodologías estándar para revisiones sistemáticas. Sin embargo es necesario enfatizar que al tratarse de una revisión enfocada en la exactitud diagnóstica de pruebas (sensibilidad y especificidad), muchas de los métodos desarrollados para las clásicas revisiones de intervenciones (enfocadas en la efectividad y seguridad de una intervención) no son aplicables en éste contexto. Un ejemplo claro de este punto es la evaluación de la heterogeneidad que los autores presentan como parte de los análisis estadísticos de la revisión. Si bien el estadístico I2 ha sido usado en extenso para la representación de la heterogeneidad, éste no es recomendado para representar la variabilidad en el escenario diagnóstico ${ }^{1}$. De igual manera, en demencia se han identificado diferentes fuentes de heterogeneidad que no deben ser ignoradas en el escenario diagnóstico, tales como los puntos de corte, el es- cenario de evaluación, los antecedentes de los pacientes, el tipo de demencia a detectar, el estándar de referencia empleado, la calidad de los estudios, la educación y el género, entre otros ${ }^{2}$. Los autores de la revisión presentan estimaciones agrupadas de la exactitud diagnóstica por prueba evaluada, las cuales ignoran la variabilidad descrita. Más aún, dichas cifras son poco informativas cuando no se identifica un determinado punto de corte para reportar la exactitud diagnóstica, lo cual está ausente en esta revisión. En la actualidad la Colaboración Cochrane se encuentra desarrollando una serie de revisiones de pruebas diagnósticas en demencia, las cuales cubren un amplio rango de instrumentos ${ }^{3-6}$. Estas revisiones han seguido los estándares más recientes respecto al desarrollo de revisiones sistemáticas de exactitud diagnóstica y permiten resolver las limitaciones que éste tipo de publicaciones presentan en su desarrollo.

\section{Conclusiones del comentador}

La presente revisión nos muestra la amplitud de la evidencia respecto a las pruebas cognitivas breves en la detección de demencia, pero falla en proporcionar información válida y útil acerca de las propiedades diagnósticas de cada una de las pruebas cognitivas evaluadas.

Ingrid Arevalo-Rodriguez [ Universidad Tecnológica Equinoccial. Facultad de Ciencias de la Salud Eugenio Espejo. Centro Colaborador Cochrane de Ecuadorinarev7@yahoo.com ]

Arevalo-Rodriguez A. El Mini Mental State Examination no es la única prueba cognitiva útil para la detección de demencia. Evid Act Pract Ambul. 2017;20(2):48. Comentado de: Tsoi KK, y col. Cognitive Tests to Detect Dementia: A Systematic Review and Meta-analysis. JAMA Internal Medicine 2015;175(9):1450-8. PMID: 26052687

\section{Referencias}

1.Macaskill P, y col. Chapter 10: Analysing and Presenting Results. In: Deeks JJ, Bossuyt PM, Gatsonis C (editors), Cochrane Handbook for Systematic Reviews of Diagnostic Test Accuracy Version 1.0. The Cochrane Collaboration, 2010. Available from: http://srdta.cochrane.org/.

2.Davis DHJ, y col. Neuropsychological tests for the diagnosis of Alzheimer's disease dementia and other dementias: a generic protocol for cross-sectional and delayed-verification studies (Protocol). Cochrane Database of Systematic Reviews 2013, Issue 3. Art. No.: CD010460. DOI: 10.1002/14651858.CD010460

3.Arevalo-Rodriguez l, y col. Mini-Mental State Examination (MMSE) for the detection of Alzheimer's disease and other dementias in people with mild cognitive impairment (MCI). Cochrane Database of Systematic Reviews 2015, Issue 3. Art. No.: CD010783. DOI: 10.1002/14651858.CD010783.pub2.

4.Fage BA, y col. Mini-Cog for the diagnosis of Alzheimer's disease dementia and other dementias within a community setting. Cochrane Database of Systematic Reviews 2015 , ssue 2. Art. No.: CD010860. DOI: 10.1002/14651858.CD010860.pub2.

5.Davis DHJ, y col. Montreal Cognitive Assessment for the diagnosis of Alzheimer's disease and other dementias. Cochrane Database of Systematic Reviews 2015, Issue 10. Art. No.: CD010775. DOI: 10.1002/14651858.CD010775.pub2.

ST, y col. Mini-Mental State Examination (MMSE) for the detection of dementia in clinically unevaluated people aged 65 and over in community and primary care populans. Cochrane Database of Systematic Reviews 2016, Issue 1. Art. No.: CD011145. DOI: 10.1002/14651858.CD011145.pub2 